\title{
Transcavernous approach to diseases of the anterior upper third of the posterior fossa
}

\author{
Ali F. KRIShT, M.D. \\ Department of Neurosurgery, University of Arkansas for Medical Sciences, Little Rock, Arkansas
}

\begin{abstract}
Object. The region in the upper anterior third of the posterior fossa is a surgically hidden, narrow corridor between the petroclival surface anteriorly and the surface of the brainstem posteriorly. Although several approaches have been described to help surgeons reach this region, few of them enable practitioners to reach the different corners of the area and provide as wide a view as the one achieved using the transcavernous route.

Methods. A transcavernous approach was used in 91 cases (50 complex upper basilar artery [BA] aneurysms, 30 upper petroclival junction meningiomas, five trigeminal nerve schwannomas, three upper clival chordomas, and three anterior pontine lesions) involving the anterior upper third of the posterior fossa. The approach uses the pretemporal route with exposure of the lateral wall of the cavernous sinus. It entails removal of the anterior clinoid process. The posterior clinoid process is also removed when necessary. The approach leads to the upper basilar region. It is widened inferiorly to expose the anterior aspect by removal of the posterior clinoid process and the petroclival osseous and dural elements. Its lateral extension exposes the region of the Meckel cave and it can be widened by removal of the petrous apex.

Seventy patients experienced new transient mild cranial neuropathies, 67 of whom recovered fully. Surgically related ischemic morbidities occurred in three patients with BA aneurysms (one small medial thalamic infarct, ataxia due to superior cerebellar artery ischemia, and distal middle cerebral artery embolus in a patient with atrial fibrillation in whom anticoagulation therapy was stopped). All the neuropathies in patients with BA aneurysms were oculomotor and recovery was the rule in all of them. Three new permanent cranial neuropathies occurred in the patients with meningiomas. In seven patients with preoperative neuropathy, two had partial improvement. Five patients with atypical meningiomas were treated with postoperative radiation therapy. Progression occurred later in four patients who were treated with gamma knife surgery. There were no surgery-related deaths. More than 1 year of follow-up data were available in 85 patients, and $94 \%$ of those patients were in an active and functional state (Glasgow Outcome Scale scores of 4 and 5 ).

Conclusions. The safety achieved with the transcavernous route allows surgeons to achieve wide exposures to lesions involving the anterior upper third of the posterior fossa. It is an approach that should be mastered by every neurosurgeon dealing with cranial lesions.
\end{abstract}

\section{KEY WORDS • posterior fossa - transcavernous approach • meningioma • basilar aneurysm • schwannoma • chordoma}

The upper third of the anterior aspect of the posterior fossa is a hidden region that is difficult to reach surgically. This is partly due to its narrow corridor between the petroclival surface anteriorly and the surface of the brainstem posteriorly, and partly due to the location of the different neurovascular structures crossing this corridor and limiting the windows through which different pathological processes can be reached. As a result, several approaches were pioneered to overcome the difficulties encountered when trying to obtain access to this difficult region. ${ }^{1,2,4-15,17-25,27-30,33,36-48}$ They all aim at providing a closer and wider access to the lesion, and at achieving a better visualization of the different vascular and neural structures.

The upper third of the anterior aspect of the posterior

Abbreviations used in this paper: $\mathrm{BA}=$ basilar artery; $\mathrm{CA}=$ carotid artery; GKS = gamma knife surgery; MR = magnetic resonance; $\mathrm{SCA}=$ superior cerebellar artery. fossa includes the following structures: 1) the interpeduncular fossa superomedially; 2) the prepontine cistern inferomedially; and 3) the region of the Meckel cave laterally. In our experience, access to all three regions can be achieved safely and effectively by using the transcavernous route. Several pathological entities involve this region, including vascular and neoplastic disease entities. ${ }^{1-3,6-18,20,23,24}$ 27-35,38,40-43,45-48 In this paper I review our group's experience with the transcavernous approach in 91 cases involving the anterior upper third of the posterior fossa and the modifications in the approach needed to provide better access to its different pathological entities.

\section{CLINICAL MATERIAL AND METHODS}

Our group used the transcavernous approach in 91 cases involving the anterior upper third of the posterior fossa (Table 1). The lesions included 50 complex upper BA an- 
eurysms, 30 meningiomas involving the upper petroclival junction region, five trigeminal nerve schwannomas, three upper clival chordomas, and three anterior brainstem lesions. There were 58 women and 33 men whose ages ranged from 17 to 67 years.

\section{SURGICAL PROCEDURE}

\section{Pretemporal Craniotomy}

The craniotomy is aimed at providing a pretemporal route similar to a pterional craniotomy but with a more temporal extension. This step is achieved by drilling the zygomatic notch to allow more inferior reflection of the temporalis muscle. The details of the pretemporal craniotomy were recently published. ${ }^{27}$ After establishing the craniotomy flap, the sphenoid wing is removed all the way medially to the level of the anterior clinoid process. The anterior clinoid process is further removed as described later (Fig. 1).

\section{Exposure of the Lateral Wall of Cavernous Sinus}

Adequate exposure of the cavernous sinus neurovascular structures help improve safety while navigating through this region. This is started by dissecting the potential plane between the dura propria of the temporal lobe and the lateral wall of the cavernous sinus. We use the meningoorbital artery as a starting point of the dissection to help establish this plane. The dura propria of the temporal lobe is sharply dissected and separated from the lateral wall of the cavernous sinus. This step achieves several advantage points.
TABLE 1

Lesions approached via the transcavernous route

\begin{tabular}{lc}
\hline \hline \multicolumn{1}{c}{ Type of Lesion } & No. of Cases \\
\hline upper BA aneurysms & $50^{*}$ \\
meningiomas of upper petroclival region & 30 \\
schwannomas of trigeminal nerve & 5 \\
chordomas of upper clivus & 3 \\
pontine lesions & 3 \\
total & 91 \\
\hline
\end{tabular}

* Of the total, 11 were SCA aneurysms.

First, it provides a better exposure of the anterior clinoid process and makes its position more superficial for its later drilling and removal. Second, it will help identify the neural elements of the cavernous sinus (except for the sixth cranial nerve) at an early stage and before starting tumor dissection. Third, in many lesions such as schwannomas and chordomas, tumor removal can be achieved extradurally.

During this phase, venous oozing from the cavernous sinus may occur and it is easily controlled by injecting Tisseal VH fiber sealant (Baxter AG, Vienna, Austria), into the different compartments of the cavernous sinus. We have used this step in more than 200 cases so far with no significant side effects. Now we routinely plan to inject Tisseal between the V1 and V2 branches of the trigeminal nerve where there is a window that leads to the main compartment

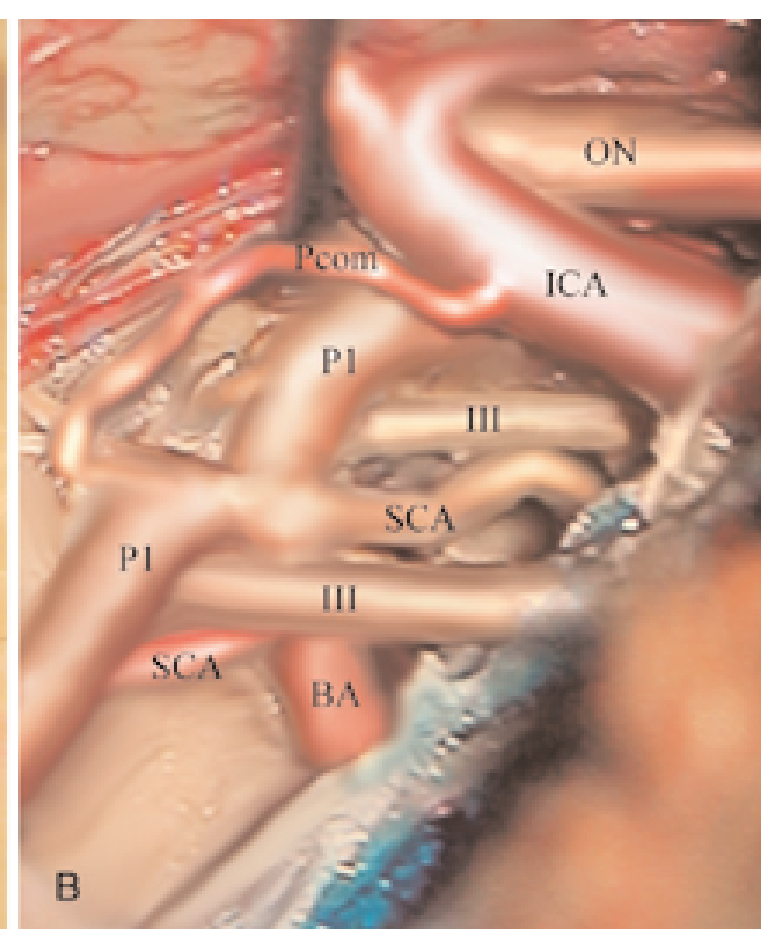

Fig. 1. A: Photograph showing the route and the extent of the exposure achieved using the transcavernous approach. The blue area highlights the more medial aspect of the approach, which is similar to what is used for basilar apex aneurysms. The pink region is the lateral extent of the approach to the region of the Meckel cave. B: A retouched anatomical photograph of the exposure achieved using the transcavernous approach. ICA = internal carotid artery; $\mathrm{ON}=$ optic nerve; Pcom = posterior communicating artery; $\mathrm{P} 1=\mathrm{P}_{1}$ segment of the posterior cerebral artery; III = oculomotor nerve. 
of the cavernous sinus. This step immediately controls cavernous sinus bleeding and minimizes epidural venous oozing. The exposure of the lateral wall of the cavernous sinus is continued posteriorly to the level of the gasserian ganglion. The exposure can be extended farther, to the level of the Meckel cave, depending on the disease entity involved. Coagulating and cutting the middle meningeal artery at the level of the foramen spinosum is a step needed to allow further posterior separation of the temporal lobe dura mater from the lateral wall of the cavernous sinus.

\section{Removal of the Anterior Clinoid Process}

The anterior clinoid process is removed by drilling and disconnecting its three main attachments to the surrounding bone structures. This includes its attachment to the roof of the optic canal, the optic strut, and its attachment to the roof of the orbit.

We prefer using a high-speed, 2- to 3-mm diamond drill to perform this step. After removal of the lateral wing of the sphenoid bone as it blends with the orbital roof, the anterior clinoid process is drilled. The drilling is directed toward the optic canal, where the drill bit is used as a sculpting tool to delineate the course of the optic nerve. Following this step, the connections of the clinoid to the optic roof and optic strut are drilled with frequent stops and copious irrigation to prevent heat buildup in the vicinity of the optic nerve. These steps, along with an already established wide exposure of the anterior clinoid process, allow gentle removal of the remaining tip of the clinoid process.

\section{Direction of the Exposure}

At this stage, additional steps are decided based on the type and location of the disease entity and its extent.

Upper BA Aneurysms. When dealing with upper BA aneurysms, the dura mater is opened in a T-shaped fashion with the vertical arm of the T along the groove of the sphenoid wing to the level of the oculomotor trigone. The third cranial nerve is visualized both intra- and extradurally and the dura mater is cut over it. This allows better mobilization of the nerve when it is dissected later in the interpeduncular fossa, and it decreases the chance of its injury.

Further exposure of the interpeduncular fossa and the prepontine cistern region is enhanced by removing the posterior clinoid process. This step is added based on the type and location of the lesion (Fig. 2). It is a step needed to establish proximal control when dealing with aneurysms arising from a low BA bifurcation (Fig. 1) Also, when dealing with brainstem lesions involving the anterior aspect of the upper pons or upper prepontine cistern, the exposure is enhanced by removal of the posterior clinoid process.

Meningiomas. In cases of meningioma, both intra- and extradural exposures may be necessary (Fig. 3). Many of the meningiomas involving this region are of the type that extends more inferiorly along the petroclival surface to where their total removal necessitates a combination of the transcavernous and petrosal approaches (Fig. 1). The dura mater is opened in a T-shaped fashion as described earlier. This allows the visualization of the whole course of the oculomotor nerve from its origin at the brainstem to the level of the superior orbital fissure. By using sharp dissection, both healthy ends of the nerve can be traced distally and proximally, leading to better and safer removal of the tumor and good preservation of the oculomotor nerve functions. The extent of our ability to remove meningiomas depends on the consistency of the tumor and the extent of its adherence to the cranial nerves and CA in the cavernous sinus. Their attachment to the intracavernous CA become more treacherous when they are dissected in patients who have undergone radiation therapy.

Schwannomas and Chordomas. In more laterally extending pathological entities, the consecutive steps are determined based on whether the main components of the tumor are located extra- or intradurally.

In case the tumor is predominantly extradural, as it is in many trigeminal nerve schwannomas (Fig. 4) or upper petroclival region chordomas (Fig. 5), there is usually no need to open the dura mater. The space left when the tumor mass is resected provides a channel allowing total removal of the tumor extradurally.

When the transcavernous route and an extradural approach are used, the anatomical details of the cavernous sinus neurovascular structures and the middle fossa osseous elements become well visualized. This makes it safe to navigate through the different cavernous sinus compartments and minimizes the possibility of inadvertent injury to the cranial nerves.

For intradural lesions, such as anterolateral pontine or prepontine lesions, the exposure is enhanced by mobilizing the trigeminal nerve at the level of the Meckel cave and drilling the petrous apex (Fig. 6).

\section{RESULTS}

Seventy patients experienced new transient mild cranial neuropathies, 67 of whom recovered fully. In BA aneurysms the most common transient neuropathy involved the oculomotor nerve, with recovery as the rule. Eleven of the aneurysms involved the SCA origin. Three patients experienced surgery-related ischemic morbidities. One patient suffered a small medial thalamic infarct resulting in a transient short-term memory deficit that had resolved in 6 months, and the patients return to full employment. The second morbidity was a transient ataxia caused by an SCA distribution ischemia. The third patient experienced a distal middle cerebral artery distribution embolism-related ischemia resulting in left upper-extremity monoparesis secondary to atrial fibrillation and discontinuation of the anticoagulation therapy in the perioperative period.

In patients with meningioma, seven presented with a progressive cranial neuropathy and improvement occurred in only two of them. Three patients experienced new permanent cranial neuropathies. In patients with meningiomas, five had atypical tumors that were treated with postoperative radiation therapy. In three of them the treatment was done using GKS. The rest of the patients were followed conservatively. The follow-up period ranges from 1 to 9 years, with a mean follow-up duration of 4.5 years. Evidence of postsurgical tumor progression occurred in four patients, all of whom were treated with GKS. All the patients with trigeminal nerve schwannomas had an excellent postoperative and long-term recovery. Two of the patients have residual minor sensory deficits in the V3 distribution, which is causing them no significant discomfort. 

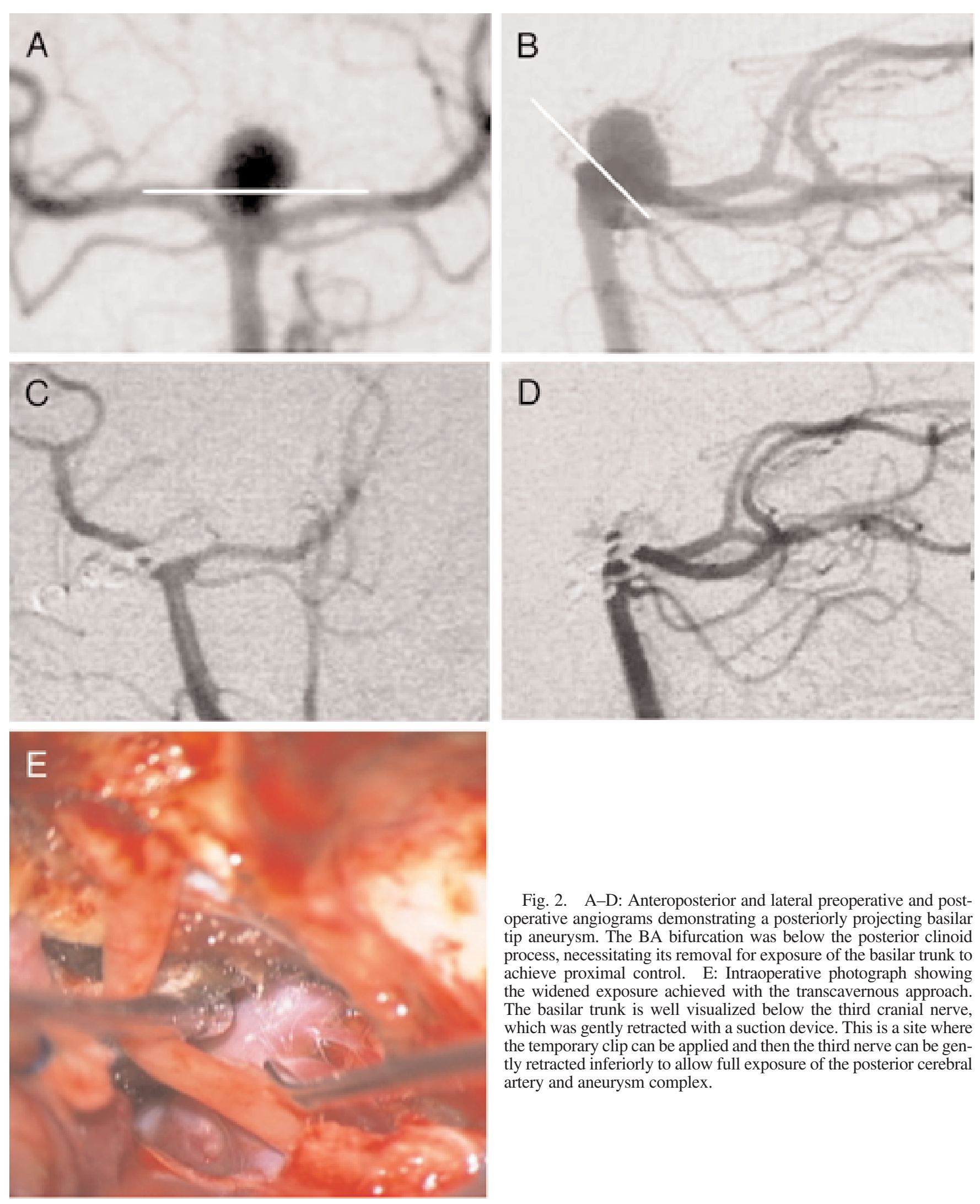

Fig. 2. A-D: Anteroposterior and lateral preoperative and postoperative angiograms demonstrating a posteriorly projecting basilar tip aneurysm. The BA bifurcation was below the posterior clinoid process, necessitating its removal for exposure of the basilar trunk to achieve proximal control. E: Intraoperative photograph showing the widened exposure achieved with the transcavernous approach. The basilar trunk is well visualized below the third cranial nerve, which was gently retracted with a suction device. This is a site where the temporary clip can be applied and then the third nerve can be gently retracted inferiorly to allow full exposure of the posterior cerebral artery and aneurysm complex. 
Corneal functions were preserved in all patients, and none had any deficits in their muscles of mastication. Their follow-up duration ranged from 1 to 5 years, with no evidence of residual or recurrent tumor.

Two of the patients with chordomas presented with progressive cranial neuropathies. In one, by the time he presented to us he had complete ophthalmoplegia. He had full recovery of his third cranial nerve functions; however, he had a residual deficit due to his abducent nerve palsy. In the other patient, who presented with a sixth cranial nerve palsy, full recovery was noted on follow-up review. All patients in whom chordoma is diagnosed undergo proton-beam radiotherapy postoperatively.

The patients with brainstem lesions have a follow-up duration ranging from 6 months to 2 years. None of the patients experienced progression. In one of the patients increased contralateral hemiparesis developed, but he recov- ered to his baseline status with rehabilitation. The other two patients have had a stable clinical course.

Other than cranial neuropathies there were three surgeryrelated permanent morbidities related to the BA aneurysms, as mentioned earlier. There were no surgery-related deaths. More than 1 year of follow-up data were available in 85 patients; 94\% of the patients were in an active and functional state (Glasgow Outcome Scale scores of 4 and 5).

\section{DISCUSSION}

In the earlier neurosurgical literature, the approaches used for the treatment of lesions involving the anterior upper third of the posterior fossa used a more lateral route. The basilar apex region, for example, was approached using the subtemporal route. ${ }^{14,15}$ When the need arises the approach was enhanced by cutting the tentorial edge. When
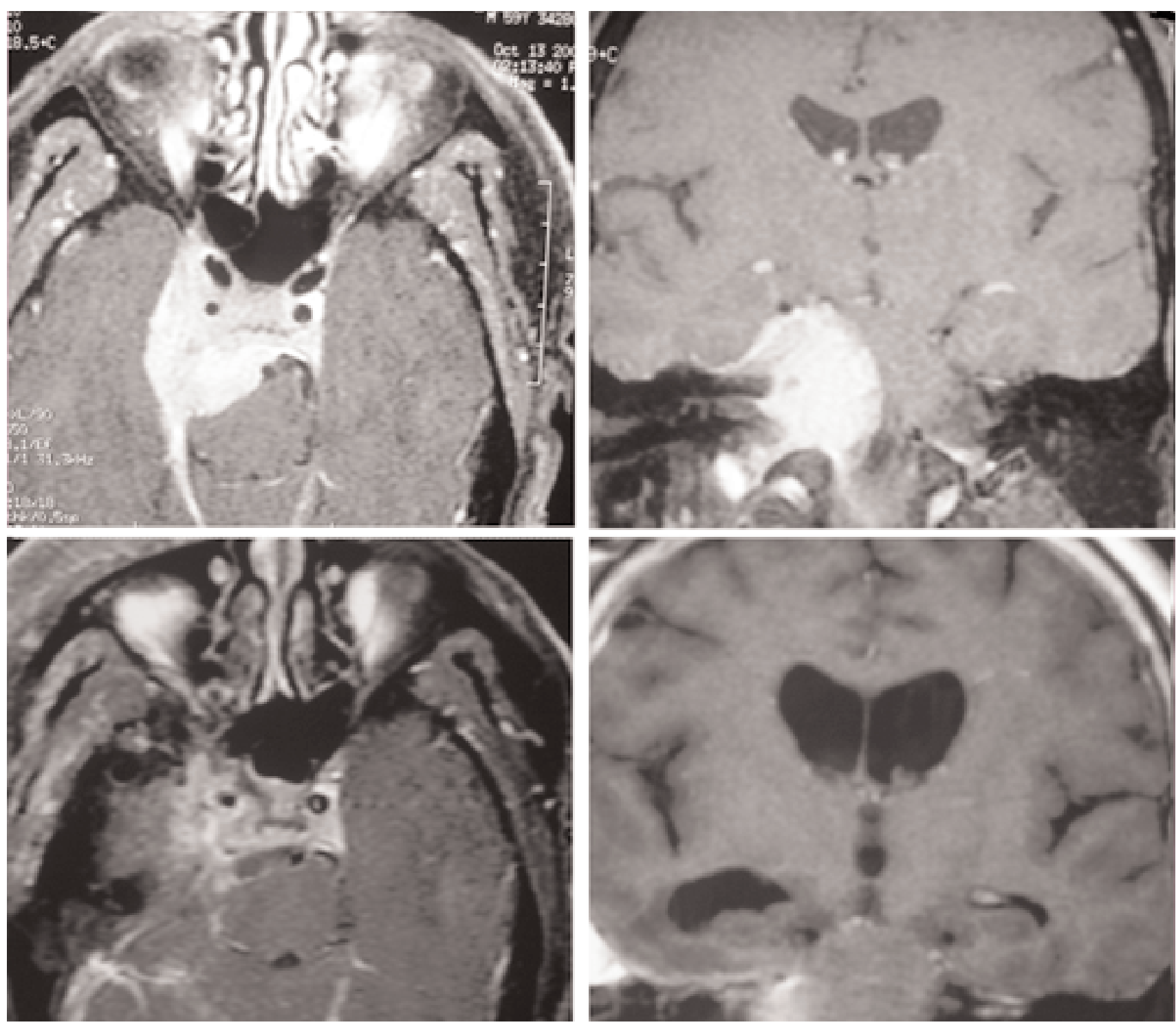

Fig. 3. Axial and coronal $\mathrm{T}_{1}$-weighted, Gd-enhanced MR images; preoperative (upper) and postoperative (lower) views. 

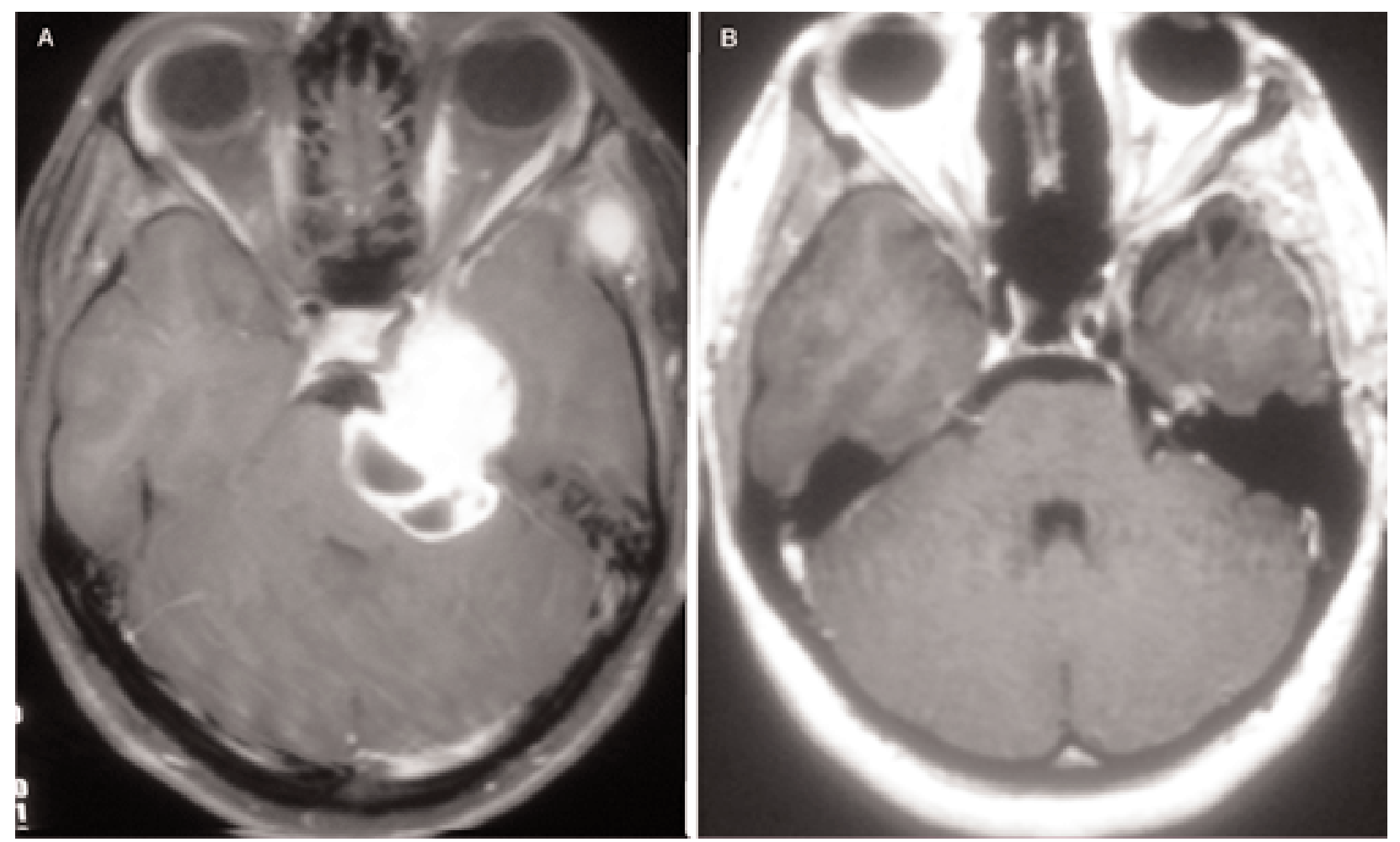

Fig. 4. Axial $\mathrm{T}_{1}$-weighted, Gd-enhanced preoperative (A) and postoperative (B) MR images of a left trigeminal nerve schwannoma involving the middle fossa and extending through the Meckel cave to the posterior fossa.

dealing with basilar apex aneurysms the limitation of the lateral approaches comes from the inability to visualize the contralateral $\mathrm{P}_{1}$. Also, in case of a low-lying BA bifurcation, the application of a temporary clip to the basilar trunk may not be easily achieved and may obstruct the view of the neck of the aneurysm. A very high bifurcation is a contraindication for the lateral approach due to the need for excessive temporal lobe retraction. Such difficulties led to the consideration of other approaches to the interpeduncular fossa; for example, the pterional approach was used as an alternative. ${ }^{45-47}$ It has its own limitations, however, in terms of the narrow space and the difficulty with proximal control in low-lying BA bifurcations.

To overcome these difficulties the transcavernous approach came as a natural evolution of the combination of our need for a wider window to this deep and hidden region, and the advances in our ability to navigate safely around the skull base microsurgical anatomy. $5,8-10,12,13,24,28,30,38$ We recently presented the advantages achieved with surgical clip occlusion of complex basilar apex aneurysms by using the transcavernous approach. ${ }^{27}$ This approach includes the following advantages: 1 ) wider exposure of the depth of the field in the interpeduncular fossa; 2) increased maneuverability during the clip application process; 3 ) the ability to apply a proximal temporary clip to a perforating vessel-free zone on the basilar trunk proximal to the SCA, preserving collateral flow to the perforating vessels of the brainstem; and 4) the temporary clip location does not obstruct the view of the neck of the aneurysm. All these advantages significantly improved the safety of clip occlusion procedures for basilar apex aneurysms.
Meningiomas involving the anterior upper third of the posterior fossa continue to present a surgical challenge. ${ }^{1,11}$, 16-18,23,33,34 The dural origin of meningiomas leads to their intra- and extradural extensions. Depending on the consistency of the tumor and the extent of its infiltration, it becomes difficult to remove these lesions totally without the risk of causing cranial neuropathies or possible vascular injury. Because of this, our policy is always to plan as radical a removal as possible as our initial goal. Our plan starts with the goal of gross-total resection. The plan is then adjusted based on the intraoperative findings and the extent of the tumor infiltration into the skull base region. The priority is to decompress the brainstem. This is then followed by the step of removing the tumor from around the neurovascular structures by sharply skeletonizing the different nerves and vascular elements. The tumor is then followed into its dural and osseous extension within the skull base.

In meningiomas it is helpful to use a neuronavigational tool to help guide the resection process. In most patients who present with a preoperative cranial neuropathy we find it more difficult to dissect the tumor from around the cranial nerves without compromising their functional recovery. In such cases, if the neuropathy is complete we proceed with aggressive tumor removal while preserving the anatomical continuity of the nerves. In patients presenting with a partial cranial neuropathy we try to decompress the nerves to enhance their functional recovery. Occasionally, this may entail leaving small islands of tumor behind, which, depending on the histopathological grading of the tumor, can be followed conservatively or be treated with focused radiation delivered using GKS. 
Transcavernous approach to anterior upper third of posterior fossa


Fig. 5. A-D: Axial and coronal Gd-enhanced MR images of a chordoma involving the cavernous sinus and sphenoid sinus region on the left side with preoperative and postoperative views. E-J: Axial, sagittal, and coronal pre- and postoperative Gd-enhanced MR images of a more medially located chordoma of the upper third of the clivus extending into the interior upper third of the posterior fossa. The tumors demonstrated in panels A through $\mathrm{D}$ and $\mathrm{E}$ through $\mathrm{J}$ were both approached via the transcavernous route, and they were removed extradurally. 

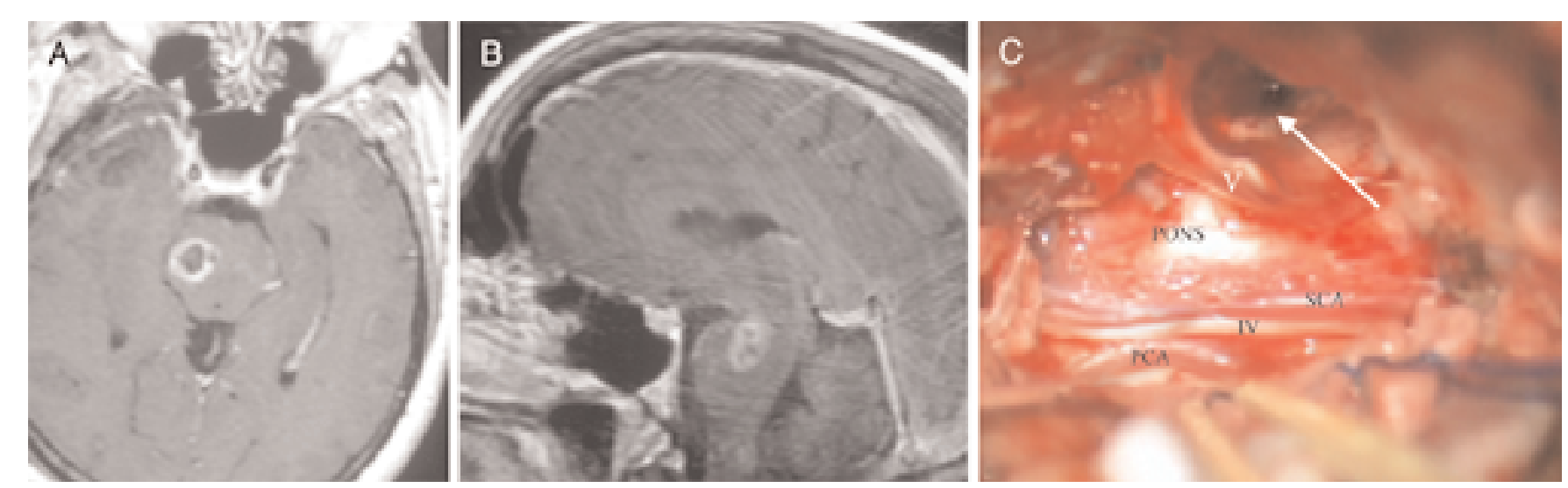

Fig. 6. A and B: Axial and sagittal MR images demonstrating a pontine lesion just posterior and inferior to the exit point of the trigeminal nerve. This astrocytic pilocytoma was approached via the transcavernous route with lateral extension to the region of the Meckel cave. $\mathrm{C}$ : Intraoperative photograph showing the view achieved through the lateral extension of the approach. The arrow indicates the pontine entry point at which the lesion reached the surface. $\mathrm{PCA}=$ posterior cerebral artery; $\mathrm{V}=$ origin of the trigeminal nerve; $\mathrm{IV}=$ trochlear nerve along the perimesencephalic cistern.

The overall majority of our patients undergo resection because of significant symptomatic brainstem compression and/or a progressive cranial neuropathy. Of the 30 patients with meningiomas reported in this paper, seven presented with progressive cranial neuropathies.

Trigeminal nerve schwannomas are easier to remove than meningiomas with the transcavernous approach. ${ }^{3,20,42,48}$ The steps of the resection are predominantly done extradurally. After making the approach, the cranial nerves are identified within the lateral wall of the cavernous sinus. The tumor is then debulked using the ultrasonic aspirator, after which the capsule is easily dissected away from the surrounding intracavernous structures. Occasionally the tumor has a significant extension into the posterior fossa. In the majority of such cases, this extension is removed by debulking the tumor and pulling the residual posterior extension into the posterior fossa space through the widened Meckel cave. The majority of these tumors arise from the V3 segment of the trigeminal nerve. Occasionally they may extend through a widened foramen ovale into the infratemporal fossa.

The postoperative recovery of patients with trigeminal nerve schwannomas resected extradurally by using the transcavernous approach proceeds remarkably well. Preservation of the trigeminal nerve functions and resolution of preoperative neuralgias is the most common scenario. $\mathrm{Pa}-$ tients are usually able to leave the hospital within a 2- to 3day period.

Generally, chordomas are better circumscribed than meningiomas. The difficulty with their excision, however, lies in the task of following the involved bone structures of the skull base. Because of the nature of the disease, it is easy to get to what seems to be the boundaries of the tumor without realizing that there are further extensions into the surrounding bone. For this reason it is important to evaluate the preoperative computerized tomography scans carefully, especially the bone windows, and to use a neuronavigational tool during the resection process.

Chordomas of the upper clival region typically extend superiorly into the suprasellar region and/or along the floor of the sella into the sphenoid sinus region. Their lateral extension is usually along the medial aspect of the Meckel cave and they tend to mobilize the intracavernous CA anteriorly and the trigeminal nerve complex laterally. The strategy for removing these tumors is first to identify and dissect the cranial nerves within the lateral wall of the cavernous sinus, followed by dissecting the intracavernous segment of the CA along its course. This is followed by debulking the tumor and careful dissection to identify the sixth cranial nerve. We believe that it is safest to find the sixth cranial nerve distally, just before its entry into the superior orbital fissure, and then follow it proximally. ${ }^{26}$ In the region of the superior orbital fissure, it is inferior to the V1 segment of the trigeminal nerve. In the majority of chordomas involving the upper clival region, extradural radical resection is possible without violating the dura mater of the posterior fossa. In this paper we only reported cases with posterior extension into the posterior fossa compartment. In upper clival chordomas that do not extend posteriorly, our preferred approach is the transsphenoidal one.

Lesions affecting the anterior aspect of the pons are fortunately not very common. Those involving the anterior or anterolateral aspect of the pons can be approached via the transcavernous route without having to remove the petrous apex. When the lesion is more laterally located, then removal of the petrous apex and mobilization of the trigeminal nerve rootlets at the level of the Meckel cave can help achieve a better exposure for such lesions. The three patients with anterior pontine lesions reported on in this series had tumors of the basis pontis. One patient had a pilocytic astrocytoma and two others had hemorrhagic lesions. In the latter two patients, findings in the pathological specimens were inconclusive in one and suggestive of a small cryptic arteriovenous malformation in the other.

The better understanding of the microsurgical anatomy of the skull base and cavernous sinus, added to further refinements in technical handling of this structure, made surgery in this region very safe. The transcavernous corridor provides a significant advantage in exposing the hidden anterior upper third of the posterior fossa. We recently extended the use of this approach to other pathological entities that may extend to the interpeduncular and prepontine cis- 
tern regions, such as retrochiasmatic craniopharyngiomas and epidermoid tumors. The transcavernous approach provides a wide exposure to a hidden and narrow region that is crowded by important neurovascular structures. It increases the maneuverability of the surgical steps and thereby significantly improves its safety. It is an approach that should be mastered by every neurosurgeon who deals with cranial lesions.

\section{ILLUSTRATIVE CASE}

This 56-year-old man presented with history of headache and a questionable subarachnoid hemorrhage from a diagnosed basilar tip aneurysm. The aneurysm was posteriorly projecting with a low-lying BA bifurcation behind the posterior clinoid. The pretemporal transcavernous approach was used, with drilling of the posterior clinoid process. Video 1 contains the preoperative angiogram, steps of the exposure and aneurysm clip occlusion, and the postoperative angiogram.

Video 1. Video of the pre- and postoperative angiogram, illustrating steps of the exposure and aneurysm clip occlusion. (Click here to view with Windows Media Player, or here to view with RealPlayer.)

\section{CONCLUSIONS}

The advances in the knowledge of the microsurgical anatomy of the cavernous sinus have made cavernous sinus surgery safe. The transcavernous approach has unlocked the door to an area of the brain that has traditionally been difficult to reach. The knowledge of these approaches should be part of the armamentarium of every neurosurgeon.

\section{References}

1. Abdel Aziz KM, Sanan A, van Loveren HR, et al: Petrovclival meningiomas: predictive parameters for transpetrosal approaches. Neurosurgery 47:139-152, 2000

2. Al-Mefty O, Ayoubi S, Gaber E: Trigeminal schwannomas: removal of dumbbell-shaped tumors though the expanded Meckel cave and outcomes of cranial nerve function. J Neurosurg 96:453-463, 2002

3. Arriaga MA, Brackmann DE: Differential diagnosis of primary petrous apex lesions. Am J Otol 12:470-474, 1991

4. Arriaga MA, Gorum M: Indications and variations of transcochlear exposure of the ventral brainstem. Laryngoscope 106: 639-644, 1996

5. Aziz KM, van Loveren HR, Tew JM Jr, et al: The Kawase approach to retrosellar and upper clival basilar aneurysms. Neurosurgery 44:1225-1234, 1999

6. Bricolo AP, Turazzi S, Talacchi A, et al: Microsurgical removal of petroclival meningiomas: a report of 33 patients. Neurosurgery 31:813-828, 1999

7. Chanda A, Nanda A: Partial labyrinthectomy petrous apicetomy approach to the petroclival region: an anatomic and technical study. Neurosurgery 51:147-159, 2002

8. Couldwell WT, Fukushima T, Giannotta SL, Weiss MH: Petroclival meningiomas: surgical experience in 109 cases. J Neurosurg 84:20-28, 1996

9. Day JD, Fukushima T, Giannotta S: Cranial base approaches to posterior circulation aneurysms. J Neurosurg 87:544-554, 1997

10. Day JD, Giannotta SL, Fukushima T. Extradural temporopolar approach to lesions of the upper basilar artery and infrachiasmatic area. J Neurosurg 81:230-235, 1994
11. De Jesus O: Petrous apex region, surgical anatomy, lesions, and operative techniques. P R Health Sci J 15:107-112, 1996

12. de Oliveira, Tedeschi H, Siqueira MG, et al: The pretemporal approach to the interpeduncular and petroclival regions. Acta Neurochir (Wien) 136:204-211, 1995

13. Dolenc VV, Skrap M, Sustersic J, et al: A transcavernous-transesellar approach to the basilar tip aneurysms. Br J Neurosurg 1:251-259, 1987

14. Drake CG: The surgical treatment of aneurysms of the basilar artery. J Neurosurg 29:436-446, 1968

15. Drake CG, Peerless SJ, Hernesniemi JA: Surgery of Vertebrobasilar Aneurysms: London, Ontario Experience on 1767 Patients. New York: Springer-Verlag, 1996

16. Gacek RR: Diagnosis and management of primary tumors of the petrous apex. Ann Otol Rhinol Laryngol 84 (Suppl 18): $1-20,1975$

17. Goel A: Extended lateral subtemporal approach for petroclival meningiomas: report of experience with 24 cases. Br J Neurosurg 13:270-275, 1999

18. Goin DW: Surgical management of petrous apex meningioma. Laryngoscope 89:204-213, 1979

19. Hsu FP, Anderson GJ, Dogan A, et al: Extended middle fossa approach: quantitative analysis of petroclival exposure and surgical freedom as a function of successive temporal bone removal by using frameless streotaxy. J Neurosurg 100: 695-699, 2004

20. Inoue T, Fukui M, Matsushima T, et al: Neurinoma in the cavernous sinus: report of two cases. Neurosurgery 27:986-990, 1990

21. Jian FZ, Santoro A, Innocenzi G, et al: Frontotemporal orbitozygomatic craniotomy to exposure the cavernous sinus and its surrounding regions. Microsurgical anatomy. J Neurosurg Sci 45:19-28, 2001

22. Kanzaki J, Kawase T, Sano K, et al: A modified extended middle fossa approach for acoustic tumors. Arch Otorhinolaryngol 217:119-121, 1977

23. Kawase T, Shiobara R, Toya S: Middle fossa transpetrosaltranstentorial approaches for petroclival meningiomas. Selective pyramid resection and radicality. Acta Neurochir (Wien) 131:315, 1994

24. Kawase T, Toya S, Shiobara R, et al: Transpetrosal approach for aneurysms of the lower basilar artery. J Neurosurg 63: 857-861, 1985

25. Knosp E, Tschabitscher M, Matula C, et al: Modifications of temporal approaches: anatomical aspects of a microneurosurgical approach. Acta Neurochir Suppl 53:159-165, 1991

26. Krisht AF, Basnett DW, Barrow DL, et al: The blood supply of hte intracavernous cranial nerves: an anatomic study. Neurosurgery 34:275-279, 1994

27. Krisht AF, Kadri PA: Surgical clipping of complex basilar apex aneurysms: a strategy for successful outcome using the pretemporal transzygomatic transcavernous approach. Neurosurgery 56:261-273, 2005.

28. MacDonald JD, Antonelli P, Day AL: The anterior subtemporal, medial transpetrosal approach to the upper basilar artery and ponto-mesencephalic junction. Neurosurgery 43:84-89, 1998

29. Megerian CA, Chiocca EA, McKenna MJ, et al: The subtemproal-transpetrous approach for excision of petroclival tumors. Am J Otol 17:773-779, 1996

30. Mortini P, Mandelli C, Franzin A, et al: Surgical excision of clival tumors via the enlarged transochlear approach. Indications and results. J Neurosurg Sci 45:127-140, 2001

31. Muckle RP, De La Cruz A, Lo WM: Petrous apex lesions. Am J Otol 19:219-225, 1998

32. Ozveren MF, Uchida L, Aiso S, Kawase T: Meningovenous structures of the petroclival region: clinical importance for surgery and intravascular surgery. Neurosurgery 50:829-836, 2002

33. Roberti F, Sekhar LN, Kalavakonda C, et al: Posterior fossa meningiomas: surgical experience in 161 cases. Surg Neurol 56:8-21, 2001 
34. Samii M, Carvalho GA, Tatagiba M, et al: Surgical management of meningiomas in Meckel's cave. Neurosurgery 41: 767-774, 1997

35. Sanna M, Mazzoni A, Saleh EA, et al: Lateral approaches to the median skull base through the petrous bone: the system of the modified transcochlear approach. J Laryngol Otol 108: 1036-1044, 1994

36. Sekhar LN, Burgess J, Akin O: Anatomical study of the cavernous sinus emphasizing operative approaches and related vascular and neural reconstruction. Neurosurgery 21:806-816, 1987

37. Sen CN, Sekhar LN: The subtemporal and preauricular intratemporal approach to intradural structures ventral to the brain stem. J Neurosurg 73:345-354, 1990

38. Seone E, Tedeshi H, de Oliveira E, et al: The pretemporal transcavernous approach to the interpeduncular and prepontine cisterns: microsurgical anatomy and technique application. Neurosurgery 46:891-898, 2000

39. Sepehrnia A, Knopp U: The combined subtemporal-suboccipital approach: a modified surgical access to the clivus and petrous apex. Minim Invasive Neurosurg 45:102-104, 2002

40. Solomon RA, Stein BM: Surgical approaches to aneurysms of the vertebral and basilar arteries. Neurosurgery 23:203-208, 1998

41. Spallone A, Makhmudov UB, Mukhamedjanov DJ, et al: Petroclival meningioma. An attempt to define the role of skull base approaches in their surgical management. Surg Neurol 51:412-420, 1999

42. Taha JM, Tew JM Jr, van Loveren HR, et al: Comparison of conventional and skull base surgical approaches for the excision of trigeminal neurinomas. J Neurosurg 82:719-725, 1995

43. Tatagiba M, Samii M, Matthies C, et al: Management of petroclival meningiomas: a critical analysis of surgical treatment. Acta Neurochir Suppl 65:92-94, 1996

44. Villavicencio AT, Leveque JC, Bulsara KR, et al: Three-dimensional computed tomographic cranial base measurements for improvement of surgical approaches to the petrous carotid artery and apex regions. Neurosurgery 49:342-352, 2001

45. Yaşargil MG: Microneurosurgery: Clinical Considerations, Surgery of the Intracranial Aneurysms and Results. Stuttgart: Georg Thieme, 1984, pp 232-295

46. Yaşargil MG: Microneurosurgery: Microsurgical Anatomy of the Basal Cisterns and Vessels of the Brain. Stuttgart: Georg Thieme, Vol 1, 1984

47. Yaşargil MG, Antic J, Laciga R, et al: Microsurgical pterional approach to aneurysm of the basilar bifurcation. Surg Neurol 6:83-91, 1976

48. Zhou L, Ren L, Li S, Guo H: Surgical treatment of trigeminal neurinomas. Chin Med J (Engl) 112:269-272, 1999

Manuscript received June 28, 2005.

Accepted in final form July 18, 2005.

Address reprint requests to: Ali F. Krisht, M.D., Department of Neurosurgery, University of Arkansas for Medical Sciences, 4301 West Markham, Slot 507, Little Rock, Arkansas 72205. email: krishtali@uams.edu. 